\title{
A 3D STOCHASTIC MODEL FOR GEOMETRICAL CHARACTERIZATION OF PARTICLES IN TWO-PHASE FLOW APPLICATIONS
}

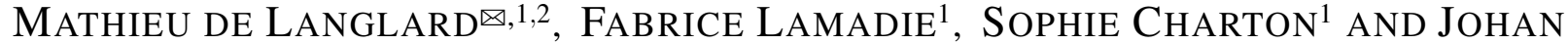 \\ DEBAYLE ${ }^{2}$ \\ ${ }^{1}$ CEA, DEN, Research Department on Mining and Fuel Recycling Processes, SA2I, F-30207 Bagnols-sur-Cèze, \\ France; ${ }^{2}$ École Nationale Supérieure des Mines, SPIN/LGF UMR CNRS 5307, Saint-Étienne, France \\ e-mail: mathieu.delanglard@gmail.com, fabrice.lamadie@cea.fr, sophie.charton@cea.fr, debayle@emse.fr \\ (Received May 3, 2018; revised September 4, 2018; accepted September 11, 2018)
}

\begin{abstract}
In this paper a new approach to geometrically model and characterize 2D silhouette images of two-phase flows is proposed. The method consists of a $3 \mathrm{D}$ modeling of the particles population based on some morphological and interaction assumptions. It includes the following steps. First, the main analytical properties of the proposed model - which is an adaptation of the Matérn type II model - are assessed, namely the effect of the thinning procedures on the population's fundamental properties. Then, orthogonal projections of the model realizations are made to obtain $2 \mathrm{D}$ modeled images. The inference technique we propose and implement to determine the model parameters is a two-step numerical procedure: after a first guess of the parameters is defined, an optimization procedure is achieved to find the local minimum closest to the constructed initial solution. The method was validated on synthetic images, which has highlighted the efficiency of the proposed calibration procedure. Finally, the model was used to analyze real, i.e., experimentally acquired, silhouette images of calibrated polymethyl methacrylate (PMMA) particles. The population properties are correctly evaluated, even when suspensions of concentrated monodispersed and bidispersed particles are considered, hence highlighting the method's relevance to describe the typical configurations encountered in bubbly flows and emulsions.
\end{abstract}

Keywords: 3D modeling, finite point process, Matérn point process, stochastic geometry, particles size distribution, two-phase flow.

\section{INTRODUCTION}

Complex random structures are ubiquitous in many industrial applications and fields of science, from physics to biology or agronomy. Typical problems involve emulsions (Maaß et al., 2011; Lau et al., 2013), porous media (Arns et al., 2003; Biswal et al., 2009) and materials characterization (Ohser and Schladitz, 2009), biological tissues (Nagata, 2000; Mrkvička and Mattfeldt, 2011) or plant anatomy (Muthukannan and Latha, 2015; Kubínova et al., 2017). In multiphase chemical processes, in particular, it is generally desired to extract information on geometrical characteristics and on spatial distribution from 2D images of the population of particles involved. Hence in bubbly flow, geometrical characteristics such as the volume fraction or the total surface of the population of bubbles define the rate of mass transfer and mixing on which the efficiency of gas-liquid process depends. In this article, population of particles refers to a collection of individuals which are either bubbles, droplets or calibrated solid spheres.

The choice of the model that properly describes a random structure is driven by the nature of the observable data. Here, the data are assumed to be
2D binarized images, also called silhouette images (Descombes, 2011; Zafari et al., 2015), where the digitized set is composed of 0 while 1 is assigned to the image's background. A stochastic modeling approach has already been used by Kracht et al. (2013) to characterize bubble images. The authors proposed a planar Boolean model of disks with a specific inference technique to retrieve the bubble size distribution. However, the population of bubbles they studied was rather diluted with little or no overlapping objects. Here, the populations studied are dense and their 2D silhouette images correspond to orthogonal projections of the components. Since an orthogonal projection of an opaque body is a many-toone mapping, the projected planar image of the body results in a loss of information. The situation is even worse when other bodies, whose projections may nontrivially overlap in the image, are added (Baddeley and Jensen , 2004). This is typically the case for twophase flow images (de Langlard et al., 2018) for which classical stereological or image analysis methods fail to retrieve $3 \mathrm{D}$ geometrical characteristics.

A different approach is proposed here that can be divided into two steps. First, a 3D marked point process model that is representative of the population 
of particles is built, while making a priori realistic assumptions. The main assumptions made are on the spherical shape of the particles, and on the type of interaction between them. Thus, the marks correspond to the radius of the particles. Besides, as in most practical cases the particles are confined in a vessel and interact with its walls, it is more consistent to incorporate the interaction of the domain $W$ with the point process in the designed model. Second, the reliability of the model is evaluated by comparing 2D synthetic images (also called modeled images in this paper) - obtained by orthogonal projection of its $3 \mathrm{D}$ realizations - and the corresponding ground truth silhouette images. From a theoretical viewpoint, the projected point process is well defined since the length $l$ of $W$ in the projection direction is finite. Note that it would not have been the case for any other homogeneous point processes, as, e.g., the homogeneous Poisson point process in $\mathbb{R}^{2}$ (its projection onto the $x$-axis is everywhere dense on any bounded interval).

The present paper is organized as follow. In the first section, the experimental materials and methods used to acquire silhouette images of the two-phase flows are detailed. The theoretical basis of the model, namely the Matern point process, and its basic properties are reminded, and the proposed methodology to model 3D populations of particles is described. Typical results are given in the second section. The sensitivity to the model parameters is tested, and the model predictions and experimental (i.e., real) images are compared. These results are further discussed in the last section.

\section{MATERIALS AND METHODS}

\section{EXPERIMENTAL SETUP}

Suspensions of solid particles are particularly convenient to acquire validation data: they can mimic the main features of any two-phase flow, while avoiding the coalescence and breakage events frequently encountered with bubbles and droplets. Using solid particles hence enables a precise knowledge of the dispersed phase properties. In this study, mixtures of calibrated PMMA particles (purchased from Goodfellow ${ }^{\mathrm{TM}}$ ) and brine are considered. The salt concentration is tuned to adjust the liquid and the solid densities in order to prevent buoyancy effects. Two populations of spherical particles are constructed: a monodipserse population of radius $r=0.75 \mathrm{~mm}$ and a volume fraction (in the liquid) of $1.5 \%$, and a bidisperse population of radius $r_{1}=0.5 \mathrm{~mm}$ and $r_{2}=0.75 \mathrm{~mm}$ with a volume fraction of $2.24 \%$. The proportion of particles of radius $r_{1}$ is equal to $63 \%$ of the total number of particles. The uncertainty on the radius given by the supplier is of $\pm 0.025 \mathrm{~mm}$. The suspensions are enclosed in a stirred tank provided with perfectly plane walls.

The optical setup is composed of a green collimated light-source and a CMOS camera (1.1 MPixel, 12 bits) from the brand Photron ${ }^{\mathrm{TM}}$, model Fastcam SA3 60K, associated to a camera lens Carl Zeiss $^{\mathrm{TM}}$, model Makro-Planar T* $100 \mathrm{~mm} f / 2$. The spatial resolution of the images is $1024 \times 1024$ where $100 \mathrm{px}$ is equal to $3.62 \mathrm{~mm}$. The depth of the tank along the optical axis is $4.5 \mathrm{~cm}$. Acquisitions are conducted at $50 \mathrm{~Hz}$, or frame per seconds (fps), with an exposure time of $1 / 30000 \mathrm{~s}$. The optical setup is calibrated with the camera placed in such a way that the middle of the tank belongs to its object focal plane. Due to a high depth of field, the whole volume of measure can be recorded without significant blur. A photograph of the experimental setup and a typical acquired image are shown in Fig. 1.

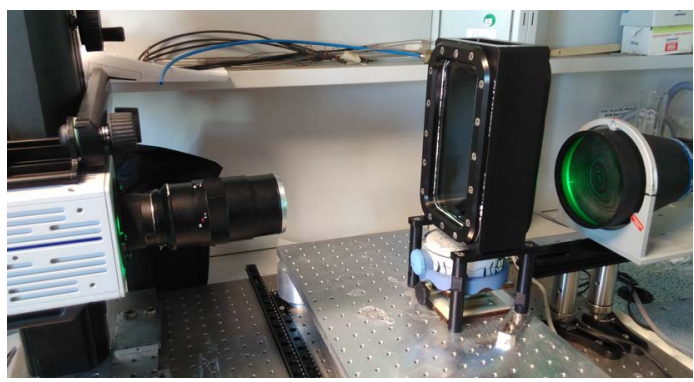

(a) Photograph of the image acquisition setup.

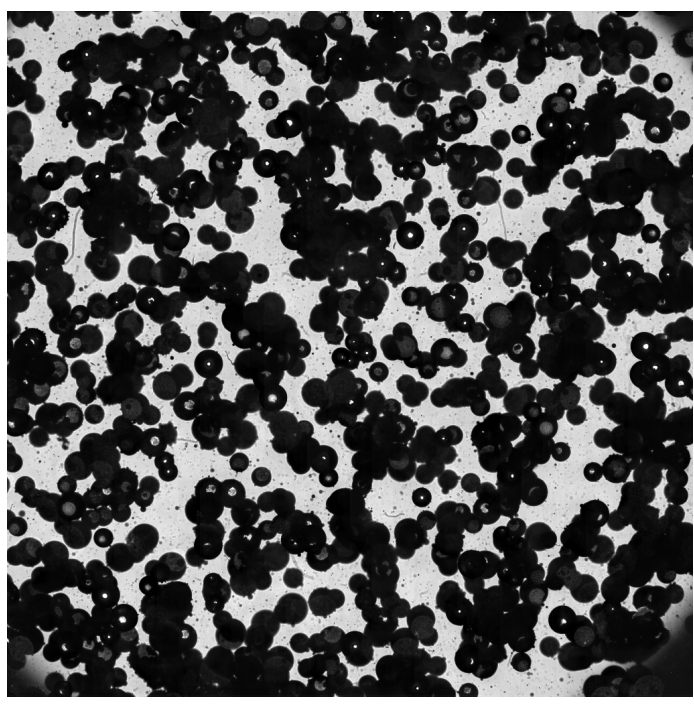

(b) Image of a suspension of calibrated PMMA spheres in water.

Fig. 1. Photograph of the experimental setup and of the two-phase flow. 
As the images are very contrasted with sharp transition between the background and the region of interest, no specific pre-processing is required. A crop of the images to remove the window effect on each corner, and a classical thresholding using Otsu's method are used. A final process is applied to remove very small objects corresponding to the presence of air bubbles stuck on the tank glass or dust. A binarized image of a calibrated population of the mono-disperse, resp. bi-disperse, population of PMMA spheres is shown in Fig. 11 (a), resp. in Fig. 13 (a) . The final size of the window of observation is $29.3 \times 37 \times 45 \mathrm{~mm}^{3}$.

\section{MODELING THE SILHOUETTE IMAGES}

The principle of the modeling approach is illustrated in Fig. 2. The 3D domain $W$ is a stripe of $\mathbb{R}^{3}$ and is denoted by $W=\mathbb{R}^{2} \times[0, l]$, where $l$ is its length along the projection direction. Within it, an observation window, $W_{\mathrm{obs}}$, is considered. It is a rectangular cuboid of length $l$ along the projection direction.

Briefly, the proposed methodology is the following:

1. Build a 3D model of the population. A hardcore spheres model is considered in this study. Besides the simplification it implies, this model is consistent with most physical problems. Similarly to particle-particle interactions, particles-wall interactions are modeled by the hard-core interaction too ;

2. Achieve its orthogonal projection on a $2 \mathrm{D}$ plane, representing the sensor plane and evaluate the properties of the obtained 2D modeled image. Just as real silhouette images, the modeled image is considered as a realization of an isotropic homogeneous random closed set, meaning invariance under rotation and translation of the distributional properties of the random set ;

3. Compare and adjust the modeled and real images using functional characteristics of random sets. The model parameters are then determined by numerical optimization.

In the following, the first two steps are described under the "Building the 3D model" subsection, whereas the last step is discussed in the "Model calibration" one.

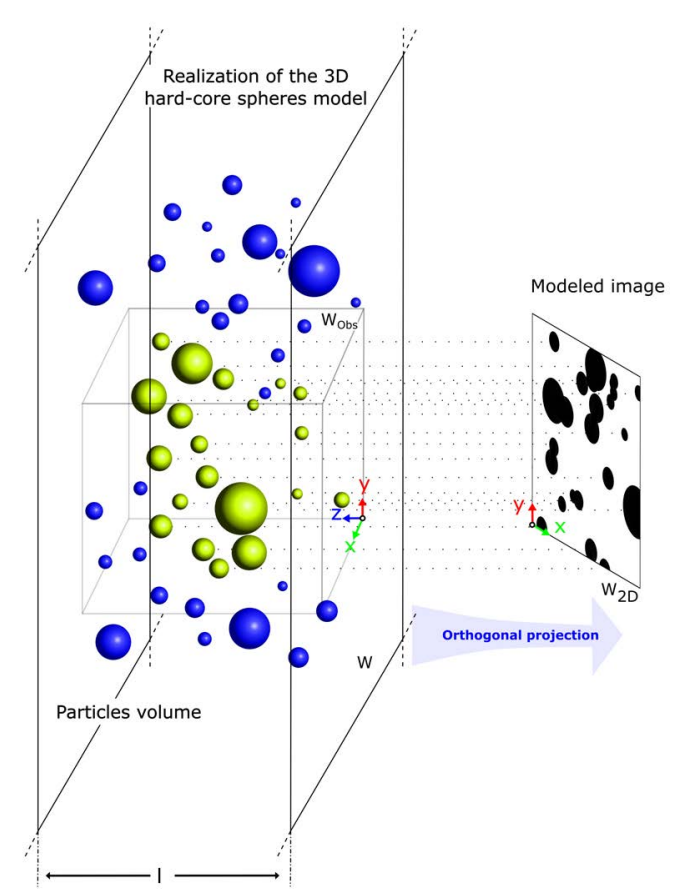

Fig. 2. Representation of the silhouette images modeling. A 3D model of hard-core spheres is chosen and orthogonal projections of its realizations are constructed to model the silhouette images of twophase flows.

\section{Reminder on Matérn's type II point process}

Matérn's type II point process (Matérn, 1960) is a thinning of an underlying homogeneous Poisson point process in $\mathbb{R}^{d}$ of intensity $\lambda$. The thinning rule introduces a hard-core distance, denoted by $R$, between the resulting points: if two points are closer than $2 R$, the last arrived one is removed. The time of arrival of a point is usually modeled using a uniform random variable taking values on the unit interval. Such a process can be considered as a marked Poisson point process, where the first mark is $R$ (constant and positive) and the second mark stands for the time of arrival.

Matérn's model can be generalized to a non deterministic mark assuming that $R$ follows a probability law. This generalization provides more flexibility in view of practical applications. If $F$ is the distribution function of the random mark $R$, it has been shown by Stoyan and Stoyan (1985) that the retention probability $g$ of a point in $\mathbb{R}^{d}$ with mark $r$ can be expressed by:

$$
g(r)=\frac{1-\exp \left(-\lambda \kappa \int_{0}^{+\infty}(r+y)^{d} F(\mathrm{~d} y)\right)}{\lambda \kappa \int_{0}^{+\infty}(r+y)^{d} F(\mathrm{~d} y)},
$$


with $\kappa$ the volume of the unit ball in $\mathbb{R}^{d}$.

This retention probability also enables to derive explicitly the intensity of the Matern point process, denoted $\lambda_{a t}$, i.e., the intensity of the point process after thinning:

$$
\lambda_{a t}=\lambda \int_{0}^{+\infty} g(r) F(\mathrm{~d} r)
$$

and the probability density function (p.d.f), denoted $f_{a t}$, of the radius after thinning (Månsson and Rudemo, 2002):

$$
f_{a t}(r)=\frac{1}{\int_{0}^{+\infty} f(x) g(x) \mathrm{d} x} f(r) g(r),
$$

where $f$ is the underlying p.d.f for $F$, the continuous distribution function of the radius before thinning.

Starting from this generalization, an adaptation of the Matérn (type II) hard-core point process is proposed to address the case of interaction between particles and the boundary of $W$ denoted by $\partial W$.

\section{Building the 3D model}

\section{Definition}

By $\mathbb{R}^{3}$ we denote the Euclidean space which is equipped with the Borel $\sigma$-algebra on the usual topology. $W$ is equipped with the induced topology by the one of $\mathbb{R}^{3}$, and hence by the corresponding Borel $\sigma$-algebra. Let $(\Omega, \mathscr{B}, \mathbb{P})$ be a probability space, $(C, \mathscr{F})$ a measurable space where $C$ denotes the space of point patterns in $W$ where the number of points is finite for any bounded Borel sets, and $\mathscr{F}$ the $\sigma$-algebra generated by the mappings $\varphi \rightarrow \varphi(B)$, where $\varphi$ is a sequence of points and $\varphi(B)$ is the number of points in the bounded Borel set $B \subset W$. Then, a general point process can be seen formally as a random variable taking values in $(C, \mathscr{F})$ (Daley and Vere-Jones, 2003).

We consider first a Poisson point process of intensity $\lambda$ in $W$. For each point, a mark is generated independently and identically distributed according to the distribution function $F$ on $\mathbb{R}^{+}$.

The proposed model is a thinning of a Poisson point process of intensity $\lambda$ resulting from the two following rules:

1. first, the thinning rule of Matern's type II model is applied (see previous section): for two marked points $\left(x_{1}, r_{1}, t_{1}\right)$ and $\left(x_{2}, r_{2}, t_{2}\right)$, if $\left\|x_{1}-x_{2}\right\|_{2} \leq$ $r_{1}+r_{2}$ then the point arrived at time $\max \left(t_{1}, t_{2}\right)$ is deleted. The deletion is effective only at the end of the process ;
2. an independent second thinning rule is applied: a marked point $(x, r, t)$ located at $x$, with radius $r$ arrived at time $t$ is removed with probability $1-\exp (-U(x, r, t))$. The function $U$, called interaction function, is positive on $W \times \mathbb{R}^{+} \times[0,1]$ and takes values in $\mathbb{R}^{+} \cup\{+\infty\}$.

Hence, in the proposed methodology, the model is fully specified by three parameters: the intensity $\lambda$ of the Poisson point process, the mark distribution function $F$, and the choice of the interaction function $U$.

\section{Main Properties}

Following these thinning procedures, the mean number of points and the corresponding mark distribution function are related to the retention probability $g(x, r)$. Its definition is therefore of prime importance.

\section{The retention probability}

The analytical expression for the retention probability $g$ is given in Proposition 1 below:

Proposition 1 The retention probability $g(x, r)$ of the point located at $x \in W$ with radius $r$ is given by:

$$
\begin{aligned}
g(x, r)= & \int_{0}^{1} \exp (-(U(x, r, t)+ \\
& \left.\left.\lambda t \int_{0}^{\infty} v\left(B_{x}(r+y) \cap W\right) F(\mathrm{~d} y)\right)\right) \mathrm{d} t,
\end{aligned}
$$

where $B_{x}(r+y)$ denotes the ball centered at $x$ and of radius $r+y$ and $v($.$) is the Lebesgue measure.$

Proof We write $\mathbb{X}:=W \times \mathbb{R}^{+} \times[0,1]$. The underlying marked Poisson point process taking values in $\mathbb{X}$ can be seen as an inhomogeneous Poisson point process of intensity measure $d \Lambda:=\lambda \mathrm{d} x F(\mathrm{~d} y) \mathrm{d} t$.

For any point $(x, r, t)$ of $\mathrm{X}$, the process of points that wins over the point $(x, r, t)$ is also an inhomogeneous Poisson point process as a thinning of an inhomogeneous Poisson point process. Its intensity measure can be expressed by

$$
\lambda \mathbb{1}\{s \leq t\} \mathbb{1}\left\{z \in B_{x}(r+y) \cap W\right\} \mathrm{d} z F(\mathrm{~d} y) \mathrm{d} s .
$$

Then, the total number of points follows a Poisson distribution of expectation

$$
\lambda t \int_{0}^{+\infty} v\left(B_{x}(r+y) \cap W\right) F(\mathrm{~d} y) .
$$

Hence, the probability that a point $(x, r, t)$ is retained after the thinning process equals the probability that there is no point of the inhomogeneous Poisson point process of expectation in Eq. 6, which is

$$
\exp \left(-\lambda t \int_{0}^{+\infty} v\left(B_{x}(r+y) \cap W\right) F(\mathrm{~d} y)\right) .
$$


We now introduce the interaction function $U$ and the corresponding probability $\exp (-U(x, r, t))$ that the point $(x, r, t)$ is retained due to this interaction. Multiplying the retaining probability related to the particle-particle interactions, Eq. 7, by $\exp (-U(x, r, t))$, provides the retaining probability accounting for the complete thinning process. Integrating over the time, it reads

$$
\begin{aligned}
g(x, r)= & \int_{0}^{1} \exp (-(U(x, r, t)+ \\
& \left.\left.\lambda t \int_{0}^{\infty} v\left(B_{x}(r+y) \cap W\right) F(\mathrm{~d} y)\right)\right) \mathrm{d} t .
\end{aligned}
$$

\section{Choice of the interaction function $U$}

The choice of the interaction function is motivated by two main reasons:

1. to simplify the formula for the retention probability of Eq. 4;

2. to verify the hardcore hypothesis for the particleswall interaction in order to be consistent with most multiphase flow applications where studied objects (droplets, bubbles or solid particles) evolve inside a finite domain (a pipe, a tank, a reactor, etc.).

In this aim, we define the hard-core particle-wall interaction function $U$, such as

$U(x, r, t)=\left\{\begin{array}{lr}+\infty, & \text { if } B_{x}(r) \cap W^{C} \neq \emptyset, \\ \lambda t \int_{0}^{+\infty} v\left(B_{x}(r+y) \cap W^{C}\right) F(\mathrm{~d} y), & \text { otherwise. }\end{array}\right.$

Hence, the retention probability $g(x, r)$ is equal to 0 when the ball centered at $x$ and of radius $r$ intersects $\partial W$. Otherwise, we have $g(x, r)=g(r)$ (as defined in Eq. 1) if the ball $B_{x}(r)$ is entirely included in $W$.

It is worth noting that $g(x, r)$ only depends on $r$ and on the coordinate $x_{3}$ along the projection direction $(\mathrm{Oz})$ and $r$ in this setting. Then, we rather write $g\left(x_{3}, r\right)$ instead of $g(x, r)$.

\section{Relations between the parameters before and after thinning}

It is worth mentioning that $(1 / l) \int_{0}^{l} g\left(x_{3}, r\right) \mathrm{d} x_{3}$ is the retaining probability of a point of radius $r$ randomly located in $W$, while $(1 / l) \int_{0}^{+\infty} \int_{W} g\left(x_{3}, r\right) \mathrm{d} x F(\mathrm{~d} r)$ represents the retaining probability of a random point of the underlying marked point process. Alternatively, one can exchange the integrals with Fubini's theorem.

Simplifying these probabilities with respect to the chosen $U$ gives the two corollaries:
Corollary 1 The intensity $\lambda_{a t}$ of the proposed model is

$$
\lambda_{a t}=\frac{\lambda}{l} \int_{0}^{l / 2}(l-2 r) g(r) F(\mathrm{~d} r)
$$

Corollary 2 We assume that the distribution function $F$ of the radius has a density $f$. Then, the probability density function $f_{a t}$ of the radius distribution after thinning is

$$
f_{a t}(r)= \begin{cases}0, & \text { if } r>l / 2, \\ k^{-1}(l-2 r) g(r) f(r) & \end{cases}
$$

where $k=\int_{0}^{l / 2}(l-2 r) g(r) f(r) \mathrm{d} r$ is the normalization constant.

\section{Illustration of the effect of the thinning rules}

The effect of the two thinning procedures is illustrated in Fig. 3 where the initial mark density $f$ and the one obtained after thinning $f_{a t}$ are compared. The histogram of the marks on 500 realizations of the Matérn model is also plotted to highlight the consistency between Corollary 2 and the simulations.

The intensity after thinning calculated from Eq. 10 equals 0.5090 whereas the estimated one from the simulations equals 0.5114 , hence a relative difference around $0.5 \%$.

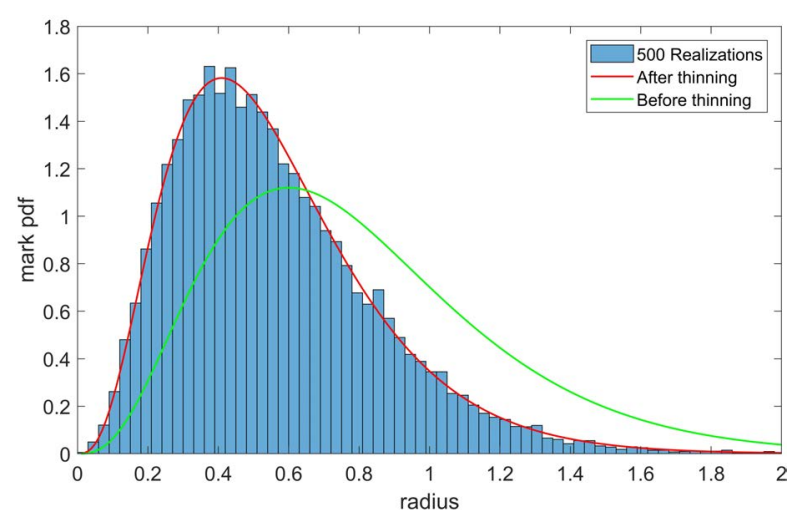

Fig. 3. Comparison of the density of the mark before and after thinning with the interaction function of Eq. 9. The length of $W$ equals $l=7$. The initial mark distribution is a gamma distribution with shape parameter $k=4$ and scale parameter $\theta=0.2$. The intensity $\lambda$ of the underlying Poisson point process equals 0.7 . 


\section{Model calibration}

It is here assumed that the model is completely described by the intensity $\lambda$ and a set of morphological parameters (the bold font is used to emphasize that $\boldsymbol{\theta}$ is a $n$-tuple and not a scalar). The aim of the calibration procedure is to infer the best values of vector $\boldsymbol{\theta}$ and of $\lambda$ such that the modeled images (i.e., resulting from the projections of the 3D model) fit the experimental images we intend to analyze. This calibration process is achieved by numerical optimization.

\section{Numerical optimization}

Several functionals can be used to characterize a region of interest in an image in terms of spatial distribution and of morphological aspect of the homogeneous random closed set to analyze. Among them, the covariance and the opening function (granulometry) are the classical statistics used (Matheron, 1972; Diggle, 1981; Dereudre et al., 2014) and have been chosen in this study.

The covariance $C$ of a random closed set $\Xi$ on $\mathbb{R}^{2}$ is defined as the following probability:

$$
\forall \boldsymbol{r} \in \mathbb{R}^{2}, \quad C(\boldsymbol{r})=\mathbb{P}\left(o \in \Xi \cap \Xi_{\boldsymbol{r}}\right),
$$

where $o$ is the origin and $\Xi_{\boldsymbol{r}}=\Xi+\boldsymbol{r}$ is the translation of $\Xi$ by the vector $r$. For a homogeneous and isotropic random set, $C$ depends only on the distance $r=\|\boldsymbol{r}\|$. An unbiased estimator $\widehat{C}$ of $C$ is:

$$
\widehat{C}(\boldsymbol{r})=\frac{v\left(\Xi \ominus\{o, \boldsymbol{r}\} \cap W_{2 D}^{\ominus r}\right)}{v\left(W_{2 D}^{\ominus r}\right)},
$$

where $v(\cdot)$ is the Lebesgue measure, $\ominus$ is the erosion operation from mathematical morphology and $W_{2 D}^{\ominus r}$ is the erosion of the bounded Borel set $W_{2 D} \subset \mathbb{R}^{2}$ by the disk of radius $r$.

Similarly, the opening function $O$ of a random closed set $\Xi$ on $\mathbb{R}^{2}$ is defined as the following probability:

$$
\forall r \geq 0, \quad O(r)=\mathbb{P}\left(o \in \Xi \circ B_{o}(r)\right),
$$

where $B_{o}(r)$ is the disk centered at the origin and of radius $r$ and $\circ$ is the classical opening operation (composition of an erosion and a dilation) of mathematical morphology. An unbiased estimator $\widehat{O}$ of $O$ is:

$$
\widehat{O}=\frac{v\left(\Xi \circ B_{o}(r) \cap W_{2 D}^{\ominus 2 r}\right)}{v\left(W_{2 D}^{\ominus 2 r}\right)} .
$$

Starting from these two unbiased estimators (Eqs. 13 and 15), the optimization problem can be formulated as the minimization of the convex combination of the $L^{2}$-norm of the model and data covariance difference on one hand, and of the $L^{2}$-norm of the model and data opening function difference on the other hand, i.e.:

$$
\begin{array}{r}
\arg \min _{\lambda, \boldsymbol{\theta}} \frac{\alpha}{\left\|\widehat{C}_{\text {data }}\right\|_{2}^{2}}\left\|\widehat{C}_{\text {model }}(\lambda, \boldsymbol{\theta})-\widehat{C}_{\text {data }}\right\|_{2}^{2}+ \\
\left.\frac{(1-\alpha)}{\left.\| \widehat{O}_{\text {data }}\right) \|_{2}^{2}} \| \widehat{O}_{\text {model }}(\lambda, \boldsymbol{\theta})-\widehat{O}_{\text {data }}\right) \|_{2}^{2},
\end{array}
$$

where $\widehat{C}_{\text {model }}(\lambda, \boldsymbol{\theta}) \quad\left(\right.$ resp. $\left.\widehat{O}_{\text {model }}(\lambda, \boldsymbol{\theta})\right)$ is the estimated average covariance (resp. average opening function) of the random set obtained by projections of the $3 \mathrm{D}$ model, $\widehat{C}_{\text {data }}$ (resp. $\widehat{O}_{\text {data }}$ ) is the estimated average covariance (resp. average opening function) of the real images, and $\alpha \in[0,1]$ is a weight parameter fixed by the user. In general, the closer to 1 is $\alpha$, the more distributional and topological information are captured by the model. Contrariwise, the closer to 0 , the more morphological aspects are retrieved by the model.

In this study, a direct search type algorithm (Davidon , 1991; Audet and Dennis, 2002) is used to find the local minimum of this optimization problem. As the space of feasible solutions is large, this optimization procedure can be very computationally time expensive. To reduce this cost, a special attention has to be paid to the the choice of the initial solution.

\section{Initialization procedure}

The steps of the initialization procedure are the following:

1. fit a $2 \mathrm{D}$ boolean model of disks on the images ;

2. deduce the $3 \mathrm{D}$ parameters after the two thinning procedures ;

3. invert the formula of Corollaries 1 and 2 to obtain an initial solution for the minimization routine.

Each of the three steps is detailed below.

\section{Fitting a 2D boolean model of disks}

The problem of finding the parameters of a boolean model of disks for a two-parameters law has been addressed by Diggle (1981); Hall (1985), and Weil (1988).

For our purpose, we use the estimation of densities method proposed by Weil (1988). Based on the area density $A_{A}$, perimeter density $L_{A}$ and Euler-Poincaré characteristics density $\chi_{A}$ of a planar Boolean model, the method of densities consists of solving a system of three equations. If we consider a disc as typical grain, 
these equations link the latter densities to the intensity $\lambda_{2 D}$ of the underlying Poisson point process, to the mean $\mu_{2 D}$ and the standard deviation $\sigma_{2 D}$ of the radius distribution. This system of equations derived by Weil (1988) for a Boolean model of discs in the plane is the following:

$$
\left\{\begin{array}{l}
A_{A}=1-q \\
L_{A}=2 \pi \lambda_{2 D} \mu_{2 D} q \\
\chi_{A}=q \times\left(\lambda_{2 D}-\frac{1}{4 \pi}\left(2 \pi \lambda_{2 D} \mu_{2 D}\right)^{2}\right)
\end{array}\right.
$$

with $q=\exp \left(-\lambda_{2 D} \pi\left(\mu_{2 D}^{2}+\sigma_{2 D}^{2}\right)\right)$. The final set of solution is denoted by $\left(\lambda_{2 D}, \mu_{2 D}, \sigma_{2 D}\right)$.

The characteristics $A_{A}, L_{A}$ and $\chi_{A}$ are estimated using the procedure of Mrkvička and Rataj (2009). Briefly, the approach of Mrkvička and Rataj (2009) consists of solving a linear regression model. The solution of this model corresponds to estimates of the intrinsic volume densities of the $\varepsilon$-parallel set of $\Xi$ (for $\varepsilon>0$ ) which can be used as approximation of those of $\Xi$ itself for small $\varepsilon$.

Alternatively, if one would like to fit a boolean model of circular grains with a non-parametric law for the radius distribution, the relatively recent method of Emery et al. (2012) using the closed relation between geometric covariogram and covariance can be used.

\section{Deduction of $\lambda_{\text {at }}$ and $f_{a t}$}

Once we have $\left(\lambda_{2 D}, \mu_{2 D}, \sigma_{2 D}\right)$, it is easy to determine the set of parameters $\left(\lambda_{a t}, \mu_{a t}, \sigma_{a t}\right)$ after thinning. The radius distribution after thinning for the proposed model is equivalent to the radius distribution of the projected marked point process (the orthogonal projection of a sphere is a disk of same radius).

Moreover, the number of particles in the observation window $W_{o b s}$ is conserved in its 2D counterpart $W_{2 D}$. The only possibility that the number of particles differs is if the projected point process in the plane is not simple, i.e. if at least two points can have the same location. This would be the case if at least two points in $W_{o b s}$ can have the same coordinate along the projection direction. However, this event has probability 0 .

Hence, the set of parameters after thinning is $\left(\lambda_{a t}, \mu_{a t}, \sigma_{a t}\right)$, with:

$$
\left\{\begin{array}{l}
\lambda_{a t}=\lambda_{2 D} / l \\
\mu_{a t}=\mu_{2 D} \\
\sigma_{a t}=\sigma_{2 D}
\end{array}\right.
$$

from which $f_{a t}$ can be deduced.

\section{Derivation of the initial solution}

To deduce the initial guess of $\lambda_{0}$ and $f_{0}$ from the previously calculated values of $f_{a t}$ and $\lambda_{a t}$, Eqs. 10 and 11 must be inverted. The inversion process is not straightforward as the two equations are interdependent, and involve complex generalized integrals. As a consequence, the determination of $\left(\lambda_{0}, \mu_{0}, \sigma_{0}\right)$ is achieved numerically with an optimization routine. It is moreover assumed that the probability law of the radius after thinning is the same as the one before thinning.

\section{RESULTS}

\section{SENSITIVITY ANALYSIS}

Before proceeding to real image analysis, and as several parameters are involved, the sensitivity of the proposed methodology is first investigated. Synthetic images, fully characterized, is used in this aim.

\section{Test of the inference procedure}

The robustness of the inference procedure is evaluated on projection's images of the proposed model, considering a gamma law for the radius distribution. The size of the observation window $W_{o b s}$ is fixed to $10 \times 10 \times 10$ and the model parameters to $\lambda_{\text {real }}=1, \mu_{\text {real }}=0.2$ and $\sigma_{\text {real }}=0.1$. A set of 200 realizations of synthetic images, as the one displayed in Fig. 4 is simulated, and we apply the calibration method with its three steps for the initialization procedure. Three values of the weight parameter $\alpha$ are considered: $\alpha=0, \alpha=1 / 2$, and $\alpha=1$.

First, a 2D boolean model of disks is fitted. To that end, the mean area density, the perimeter density and the Euler-Poincaré characteristic density are calculated from the whole set of images. The application of the method of densities leads to $\lambda_{2 D}=7.84, \mu_{2 D}=$ 0.211 , and $\sigma_{2 D}=0.066$ as well as the corresponding parameters after thinning $\lambda_{a t}=0.784, \mu_{a t}=0.211$ and $\sigma_{a t}=0.066$. It provides the initial solution of the optimization procedure $\lambda_{0}=1.11, \mu_{0}=0.221$, and $\sigma_{0}=0.07$, from which the three optimization routines for Eq. 16 are run. The obtained results are gathered in Table 1 where they are compared with the real ones.

For $\alpha=0$, the parameters found are very close to the true solution: a small deviation of around 5\% is obtained for the intensity, and less than $3.5 \%$ for the mean and standard deviation of the radius distribution. For $\alpha=1 / 2$ (resp. $\alpha=1$ ), the deviation from the true intensity value is around $9.5 \%$ (for both value of $\alpha$ ), about $5.5 \%$ (resp. $4 \%$ ) for the mean and $2 \%$ 
(resp. 9\%) for the standard deviation of the radius distribution. The corresponding fitted covariances and opening functions are provided in Fig. 5.

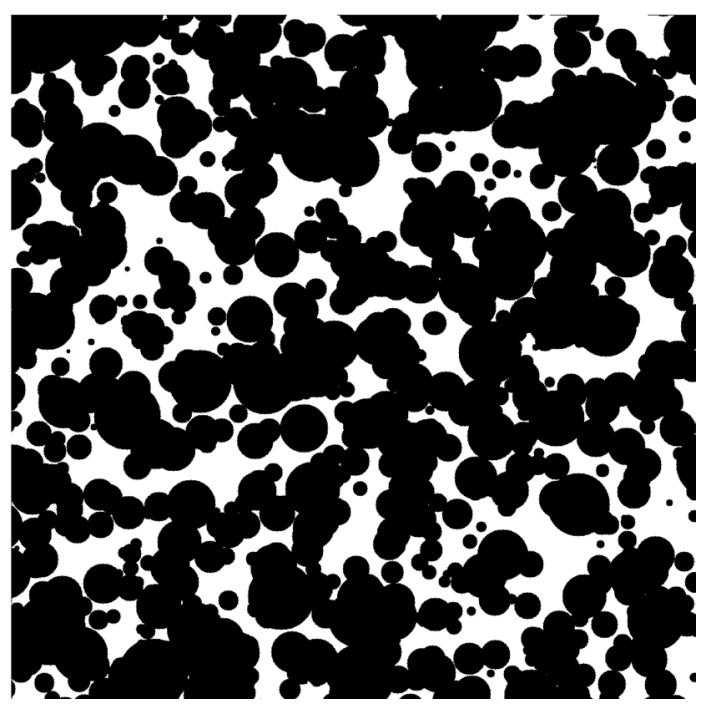

Fig. 4. Example of a synthetic image obtained by an orthogonal projection of a realization of the proposed model with intensity $\lambda_{\text {real }}=1$ and radius distribution $\Gamma(4,0.05)$.

\section{Robustness to the volume fraction}

Increasing the volume fractions of spheres in the population is considered to test the robustness of the calibration procedure.

Let $V_{V}$ be the volume fraction of the population of particles (i.e. after thinning). As we deal with noninterpenetrating particles, it follows

$$
V_{V}=\lambda_{a t} \kappa \mathbb{E}_{F_{a t}}\left[R^{3}\right],
$$

where $F_{a t}$ denotes the cumulative distribution function of the radius after thinning, $\kappa$ the volume of the unit ball of $\mathbb{R}^{3}$, and $\mathbb{E}_{F_{a t}}[$.$] the expectation under F_{a t}$.

Table 1. Results of the calibration procedure on the synthetic images when a gamma distribution is chosen both to generate the synthetic images and for the modeling approach. Three values of the weight parameter $\alpha$ have been tested: $0,1 / 2$ and 1 . The results are reported for a single optimization routine.

\begin{tabular}{cccccc}
\hline & \multirow{2}{*}{ Real } & \multirow{2}{*}{ Init } & \multicolumn{3}{c}{ Weight parameter $\alpha$} \\
& & & 0 & $1 / 2$ & 1 \\
\hline$\hat{\lambda}_{a t}$ & $\mathbf{0 . 8 1 1}$ & 0.856 & 0.770 & 0.889 & 0.898 \\
$\hat{\mu}_{a t}$ & $\mathbf{0 . 1 8 6}$ & 0.213 & 0.192 & 0.176 & 0.179 \\
$\hat{\sigma}_{a t}$ & $\mathbf{0 . 0 9 1}$ & 0.066 & 0.092 & 0.093 & 0.083 \\
\hline
\end{tabular}

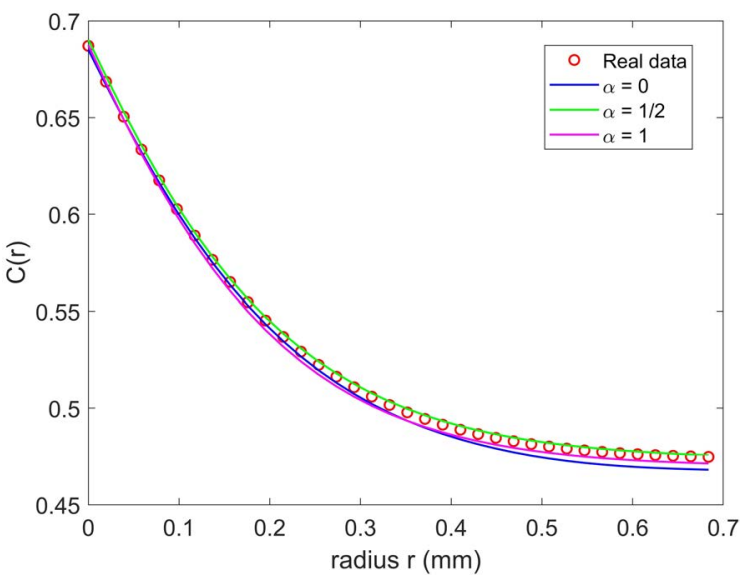

(a) Fitted covariance

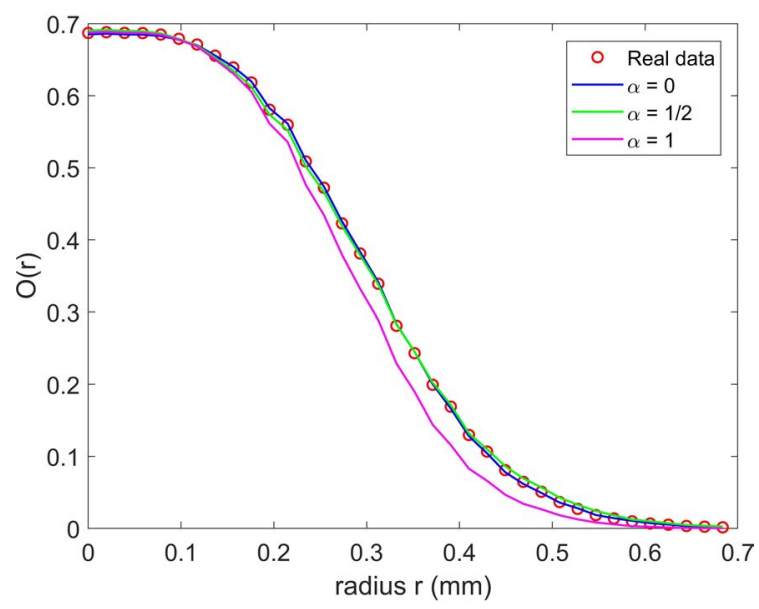

(b) Fitted opening function

Fig. 5. Comparison of the characteristics of the real data (synthetic images from the proposed model) and of the modeled images. The radius distribution used in the modeling is the same as the one to generate the images (a gamma law $\Gamma(4,0.05)$ ). The real data characteristics are plotted in red circles.

According to Eqs. 10 and 11, it reads

$$
V_{V}=\frac{1}{l} \int_{0}^{l / 2} r^{3}(l-2 r)(\lambda \kappa g(r)) f(r) \mathrm{d} r .
$$

If we consider $V_{V}$ as a function of $\lambda$, it is clear that $V_{V}$ is a non-decreasing function of $\lambda$. Moreover, taking the limit when $\lambda \rightarrow \infty$ gives:

$$
V_{V, \lim }=\frac{1}{l} \int_{0}^{l / 2} r^{3}(l-2 r) \frac{1}{\int_{0}^{+\infty}(r+y)^{3} f(r) \mathrm{d} y} f(r) \mathrm{d} r .
$$

Keeping the same radius distribution (only the initial intensity $\lambda$ is changed to impact on the volume fraction), the corresponding function $V_{V}(\lambda)$ is illustrated on Fig. 6. Notice that $V_{V}$ depends also on the mean and standard deviation of the radius distribution, which are kept constant here. 


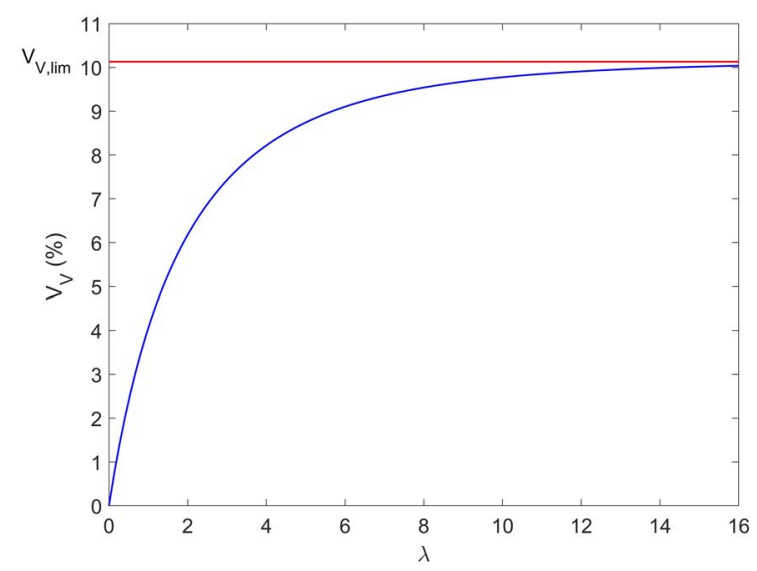

Fig. 6. The volume fraction function after thinning with respect to the intensity $\lambda$ for a gamma radius distribution with mean 0.2 and standard deviation 0.1 . The limit $V_{V, \lim }$ when $\lambda \rightarrow \infty$ equals respectively $10.13 \%$.

To assess the accuracy of the proposed method regarding the volume fraction, several values of $V_{V}$ are tested while keeping $l=10$. For each value $V_{V, i}$, a set of synthetic images is generated and the calibration procedure is tested on each set. To account for the sampling effects (due to the estimation of the covariance and opening function), the optimization routine is run several times (50 times in practice) for each value of $V_{V, i}$. The results are presented in Fig. 7 for $\alpha=1 / 2$.

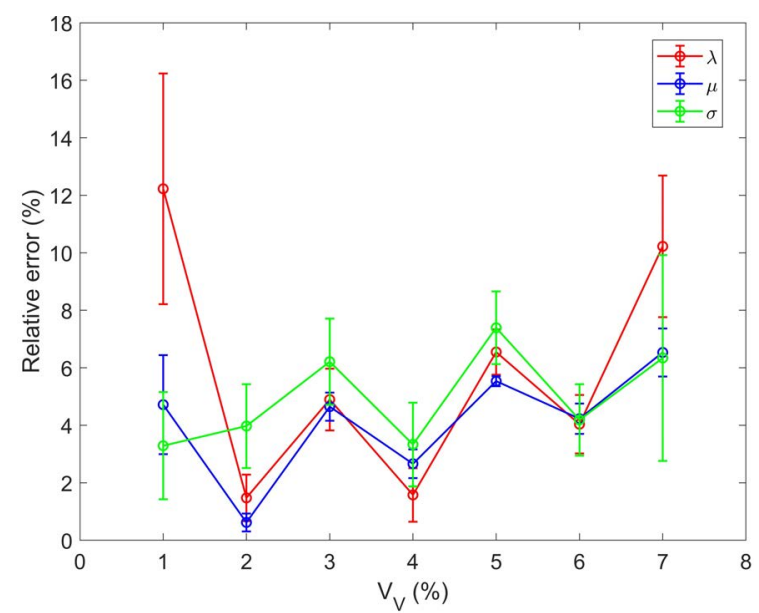

Fig. 7. Relative error of the estimated parameters for several volume fractions $V_{V}$ of particles in $W$. The gamma radius distribution has a mean of 0.2 and standard deviation of 0.1 . The circles represent the mean of the relative error for each parameter and the error bars the standard deviation for 50 simulation tests for each value of $V_{V}$.
For the considered volume fractions, the mean relative errors of the mean and the standard deviation with their counterpart are between $0.5 \%$ and $7 \%$. The same behavior is observed for the intensity except, for the volume fraction $V_{V}=1 \%$ where the mean relative error is around $12 \%$, and for $V_{V}=7 \%$ where the mean relative error is around $10 \%$. This will be discussed in the next section.

\section{Sensitivity to the initial solution}

The sensitivity of the optimization procedure to the initial solution are investigated using the same set of parameters as in the first case study, while different deviation's magnitudes for the initial solution are tested.

We can express the set of perturbed initial solution in the following form

$$
\left(\lambda_{0}^{\Delta}, \mu_{0}^{\Delta}, \sigma_{0}^{\Delta}\right)=(\lambda, \mu, \sigma) \times(1+\Delta),
$$

where $(\lambda, \mu, \sigma)$ is the theoretical set of parameters before thinning and $\Delta:=\left(\Delta_{\lambda}, \Delta_{\mu}, \Delta_{\sigma}\right)$ is the vector of perturbations for each parameter.

Here, we start from the difference of the previous constructed initial solution and the real one. In the first case study, the initial solution (before thinning) can be recovered with $\Delta_{\lambda}=\Delta_{\mu} \approx 0.1$ and $\Delta_{\sigma}=-0.3$. From this value of the vector $\Delta$, the magnitude of the perturbation is increased stepwise to find the largest value of $\Delta$ such that the algorithm still converges to a satisfactory local minimum. Therefore, several values of $\Delta$ are chosen:

$-\Delta_{1}=(0.15,0.15,-0.35) ;$
$-\quad \Delta_{2}=(0.20,0.20,-0.40) ;$
- $\Delta_{3}=(0.25,0.25,-0.45) ;$
- $\Delta_{4}=(0.30,0.30,-0.50)$.

The results of the calibration procedure for these 4 sets of perturbed initial solution are provided in Table 2, where the relative errors $\left(\lambda_{\mathrm{at}}^{\text {real }}-\widehat{\lambda}_{\mathrm{at}}\right) / \lambda_{\mathrm{at}}^{\text {real }} \times$ $100 \%$ are computed (similarly for the mean and standard deviation).

Table 2. Relative error for each parameter for several perturbed sets of the initial solution. The first row corresponds to the relative errors obtained in the first case study.

\begin{tabular}{crrr}
\hline & $\widehat{\lambda}_{a t}$ & \multicolumn{1}{c}{$\widehat{\mu}_{a t}$} & \multicolumn{1}{c}{$\widehat{\sigma}_{a t}$} \\
\hline$\left(\lambda_{0}, \mu_{0}, \sigma_{0}\right)$ & $9.5 \%$ & $-5.5 \%$ & $1 \%$ \\
$\Delta_{1}=(0.15,0.15,-0.35)$ & $4 \%$ & $-4 \%$ & $5 \%$ \\
$\Delta_{2}=(0.20,0.20,-0.40)$ & $4 \%$ & $-8 \%$ & $9 \%$ \\
$\Delta_{3}=(0.25,0.25,-0.45)$ & $3 \%$ & $-13 \%$ & $15 \%$ \\
$\Delta_{4}=(0.30,0.30,-0.50)$ & $-11 \%$ & $-5 \%$ & $20 \%$ \\
\hline
\end{tabular}


For the two smallest perturbed initial solutions $\left(\lambda_{0}^{\Delta_{1}}, \mu_{0}^{\Delta_{1}}, \sigma_{0}^{\Delta_{1}}\right)$ and $\left(\lambda_{0}^{\Delta_{2}}, \mu_{0}^{\Delta_{2}}, \sigma_{0}^{\Delta_{2}}\right)$, the maximum relative error on the set of parameters is less than $5 \%$ and $9 \%$ respectively. More significant errors are obtained for the two other perturbations.

\section{Robustness to polydispersity}

Lastly, a final test is undertaken for a radius distribution which is not unimodal, in order to check the capacity of the inference procedure to recover the characteristic radius of the spheres as well as the proportion of each sub-population.

The generated population of spheres corresponds to a mixture of $1 / 3$ of spheres with radius $r_{1}=0.5$ and of $2 / 3$ of spheres with radius $r_{2}=0.75$. The radius distribution is characterized by the following discrete probability law, i.e.,

$$
\forall r \geq 0, \quad P(r)=\beta \delta_{r_{1}}(r)+(1-\beta) \delta_{r_{2}}(r),
$$

where $\beta \in[0,1]$ corresponds to the proportion of spheres of radius $r_{1}$ before thinning.

The set of synthetic images is generated in the observation window $W_{\text {obs }}=20 \times 20 \times 20$ with intensity $\lambda=0.05, r_{1}=0.5, r_{2}=0.75$ and $\beta=1 / 3$.

Following the initialization routine, a Boolean model of disks is fit on the images giving the following set of parameters: $\lambda_{2 D}=0.895, \mu_{2 D}=0.653$ et $\sigma_{2 D}=$ -0.0001 . While the intensity $\lambda_{2 D}$ is still relevant for the initialization, the mean and the standard deviation are difficult to interpret, as here the radius distribution is neither unimodal nor continuous, and are therefore not sufficient to recover the parameters of interest $\left(r_{1,0}, r_{2,0}, \beta_{0}\right)$.

If it is possible to observe directly isolated balls from the image, then the two specific radius can be extracted and the optimization routine is used only to determine $(\lambda, \beta)$. Otherwise, if no isolated balls are observable because of the overlapping, then the initial radius $\left(r_{1,0}, r_{2,0}\right)$ have to be determined using the slope discontinuities in the opening function (Fig. 8).

The last important parameter to consider is the proportion of spheres of radius $r_{1}$ after thinning. Either $\beta_{a t}$ is known and $\beta_{0}$ can be determined analytically, or $\beta_{a t}$ is unknown and $\beta_{0}$ is chosen empirically and used in the optimization routine.

Therefore, we fix $\lambda_{0}=\lambda_{2 D} / l=0.041$ and three settings are investigated:

1. $\beta_{a t}$ is known and $\left(r_{1}, r_{2}\right)$ is unobserved:

Then, $\left(r_{1,0}, r_{2,0}\right)$ is determined with the opening function. We find $r_{1,0}=0.49 \mathrm{~mm}$ and $r_{2,0}=$ $0.72 \mathrm{~mm}$.
The proportion $\beta$ in the modeled population can be directly evaluated from the one after thinning, $\beta_{a t}$. Indeed, Eqs. 1 and 23 lead to the following retention probability:

$$
\left\{\begin{array}{l}
g\left(r_{1}\right)=\frac{1-\exp \left(-\lambda \kappa\left(\beta\left(2 r_{1}\right)^{3}+(1-\beta)\left(r_{1}+r_{2}\right)^{3}\right)\right)}{\lambda \kappa\left(\beta\left(2 r_{1}\right)^{3}+(1-\beta)\left(r_{1}+r_{2}\right)^{3}\right)} \\
g\left(r_{2}\right)=\frac{1-\exp \left(-\lambda \kappa\left((1-\beta)\left(2 r_{2}\right)^{3}+\beta\left(r_{1}+r_{2}\right)^{3}\right)\right)}{\lambda \kappa\left((1-\beta)\left(2 r_{2}\right)^{3}+\beta\left(r_{1}+r_{2}\right)^{3}\right)}
\end{array}\right.
$$

Hence, $\beta_{0}$ can be deduced from $\lambda_{0}, r_{1,0}$, and $r_{2,0}$ for

$$
\begin{gathered}
P_{a t}\left(r_{1}\right)=k^{-1}\left(l-2 r_{1}\right) g\left(r_{1}\right) P\left(r_{1}\right) \\
\Longleftrightarrow \beta_{a t}=k^{-1} \beta\left(l-2 r_{1}\right) \times \\
\frac{1-\exp \left(-\lambda \kappa\left(\beta\left(2 r_{1}\right)^{3}+(1-\beta)\left(r_{1}+r_{2}\right)^{3}\right)\right)}{\lambda \kappa\left(\beta\left(2 r_{1}\right)^{3}+(1-\beta)\left(r_{1}+r_{2}\right)^{3}\right)},
\end{gathered}
$$

where $k$ is the corresponding normalization constant.

Fixing $\beta_{0}=1 / 3$, the best result is achieved for $\alpha=0: \widehat{\lambda}=0.0482, \widehat{r_{1}}=0.521, \widehat{r_{2}}=0.731$ and $\widehat{\beta}=0.27$.

2. $\beta_{a t}$ is unknown and $\left(r_{1}, r_{2}\right)$ is unobserved:

If there is no a priori information on $\beta_{a t}, \beta$ is initialized to $1 / 2$.

For the case considered here, the best result is also obtained for $\alpha=0: \widehat{\lambda}=0.0533, \widehat{r_{1}}=0.506$, $\widehat{r_{2}}=0.751$ and $\widehat{\beta}=0.379$.

3. $\beta_{a t}$ is unknown and $\left(r_{1}, r_{2}\right)$ is observed:

By identifying isolated balls, $\left(r_{1}, r_{2}\right)=(0.5,0.75)$ are measured. It remains to determine $\lambda$ and $\beta$.

As previously, we fix $\beta_{0}=1 / 2$. The best result is obtained for $\alpha=0$ with $\widehat{\lambda}=0.046$ and $\widehat{\beta}=0.24$.

The fitted covariance and opening function obtained in the three cases for $\alpha=0$ are compared in Fig. 8 .

In each case the opening function is quite well replicated. However, a shift in the covariance is visible. For $\alpha=1 / 2$, the estimated parameters are similar for the first setting while significant deviations from the true values are noted for the two others. 


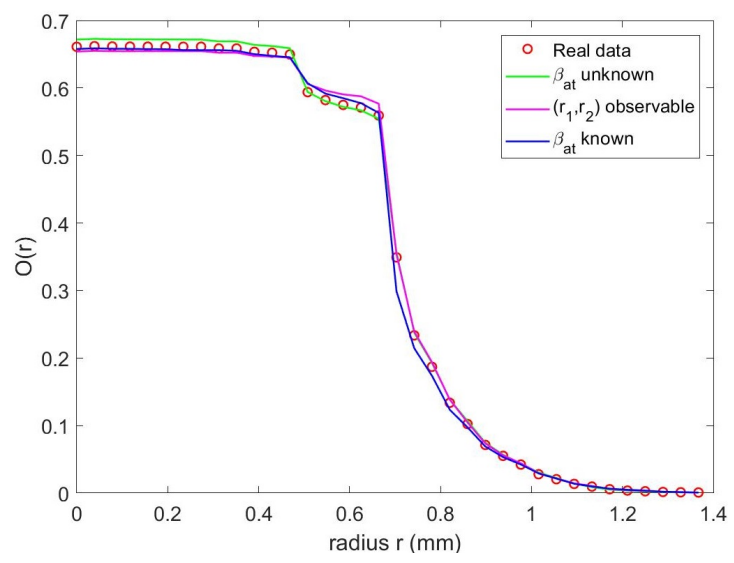

(a) Fitted opening function

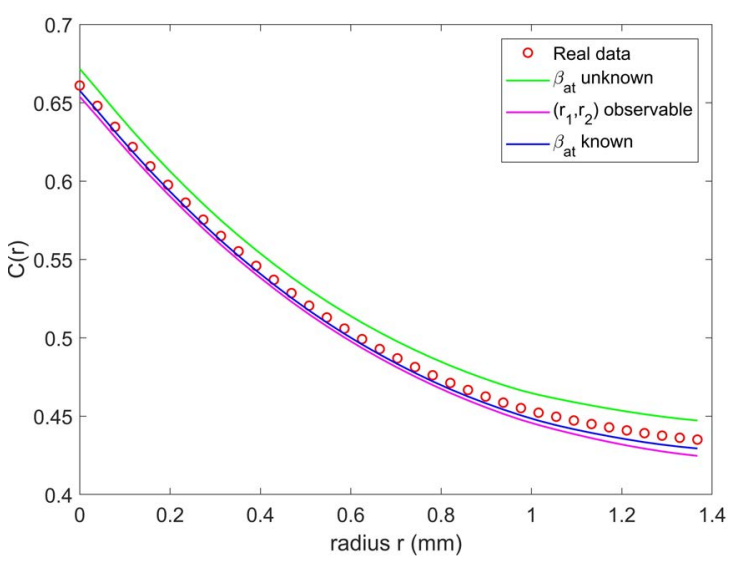

(b) Fitted covariance

Fig. 8. Characteristics of the fitted model on a set of synthetic images generated from the same model with a bi-disperse radius distribution. The result is obtained for one optimization routine.

\section{APPLICATION TO REAL IMAGES}

First experiment: monodisperse population of spheres

The population of particles is here composed of spheres of fix radius $r=0.75$. The estimated covariance in the three main directions (horizontal, diagonal and vertical) is provided in Fig. 9. The observed similarity of the covariance in the three directions validates the isotropy assumption of the considered random set.

The results obtained are provided in Table 3 assuming a constant radius distribution in the inference procedure.

The best fitting covariance and opening functions are obtained for $\alpha=0$ or $\alpha=1 / 2$ (see Fig. 10). From a geometrical point of view, a difference of $0.004 \mathrm{~mm}$ (taking into consideration the manufacturing uncertainty of $0.025 \mathrm{~mm}$ on the radius) are found. The relative error on the mean number of particles is $2.5 \%$.
Table 3. Results of the calibration procedure on the calibrated PMMA spheres when a constant radius is chosen.

\begin{tabular}{llllll}
\hline & \multirow{2}{*}{ Real } & \multirow{2}{*}{ Init } & \multicolumn{3}{c}{ Weight parameter $\alpha$} \\
& & & \multicolumn{1}{c}{$\boldsymbol{1}$} & \multicolumn{1}{c}{$1 / 2$} & \multicolumn{1}{c}{1} \\
\hline$\hat{\lambda}_{a t}$ & $\mathbf{0 . 0 0 8 5}$ & 0.0085 & 0.0087 & 0.0087 & 0.0078 \\
$\hat{r}_{1}$ & $\mathbf{0 . 7 5}$ & 0.842 & 0.779 & 0.779 & 0.842 \\
\hline
\end{tabular}

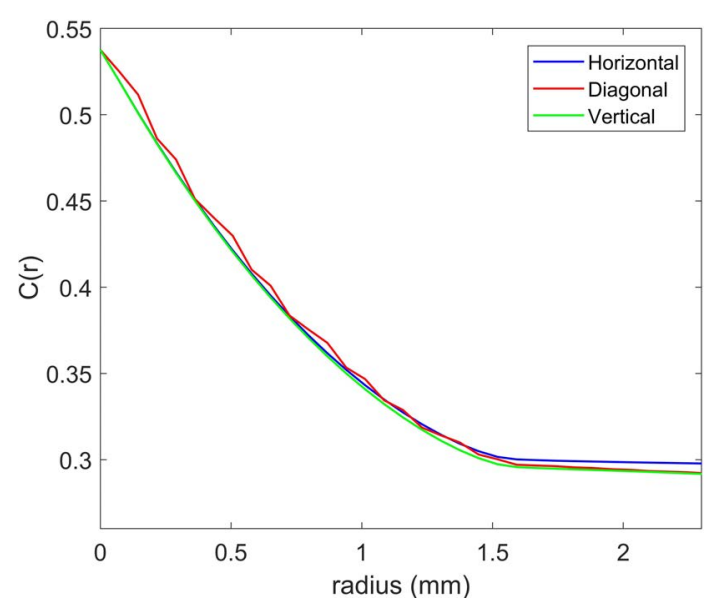

Fig. 9. Mean covariance on 100 silhouette images of the population of PMMA spheres in the three main directions.

For completeness, we give a qualitative comparison between a modeled image and a binarized experimental one in Fig. 11.

Second experiment: bidisperse population of spheres

The population of particles is here composed of two sub-populations: the first one, with a proportion $\beta=0.63$, is composed of spheres with radius $r_{1}=$ $0.5 \mathrm{~mm}$, and the second subpopulation of spheres with radius $r_{2}=0.75 \mathrm{~mm}$.

The initialization procedure leads to $\lambda_{0}=0.0196$, $r_{1,0}=0.0505, r_{2,0}=0.758$, and we fixed $\beta_{0}=1 / 2$. The results of the optimization routine are provided in Table 4. The best fitted covariance and opening function are obtained for $\alpha=1 / 2$ (Fig. 12). A comparison of the binarized experimental images and modeled images for this set of parameters is also supplied in Fig. 13. Instead, for $\alpha=1$, there is no change in the radius values from the initial solution and significant deviations of both fitted functional characteristics from the real ones are observed. 


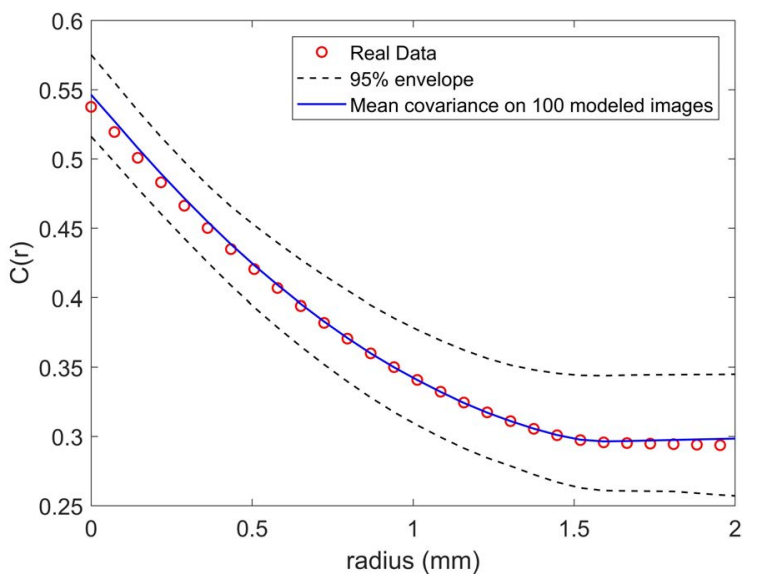

(a) Fitted covariance

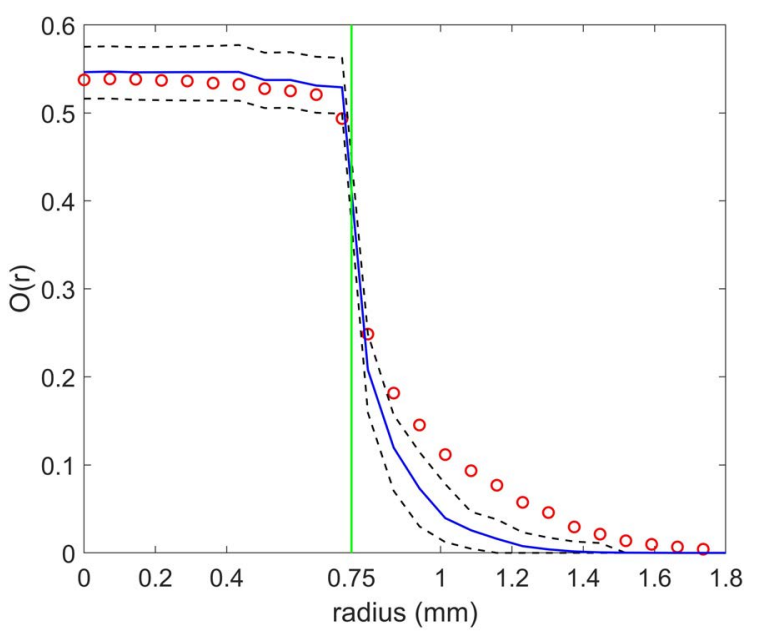

(b) Fitted opening function

Fig. 10. Fitted characteristics for the two-phase flow where the dispersed phase is a controlled population of monodispersed PMMA spheres $(r=0.75 \mathrm{~mm})$. The real data characteristics are plotted in red circles. The mean characteristics of the fitted model on a set of 100 images are represented in blue lines and its 95\% envelope in black dashed-lines.

Table 4. Results of the calibration procedure on the bi-disperse calibrated PMMA spheres population.

\begin{tabular}{lcllll}
\hline & \multirow{2}{*}{ Real } & \multirow{2}{*}{ Init } & \multicolumn{3}{c}{ Weight parameter $\alpha$} \\
& & & 0 & $1 / 2$ & \multicolumn{1}{c}{1} \\
\hline$\hat{\lambda}_{a t}$ & $\simeq \mathbf{0 . 0 2 3}$ & 0.0171 & 0.0171 & 0.0179 & 0.0186 \\
$\hat{r}_{1}$ & $\mathbf{0 . 5}$ & 0.505 & 0.579 & 0.567 & 0.505 \\
$\hat{r}_{2}$ & $\mathbf{0 . 7 5}$ & 0.758 & 0.758 & 0.742 & 0.758 \\
$\hat{\beta}_{a t}$ & $\simeq \mathbf{0 . 6 3}$ & 0.52 & 0.51 & 0.41 & 0.25 \\
\hline
\end{tabular}

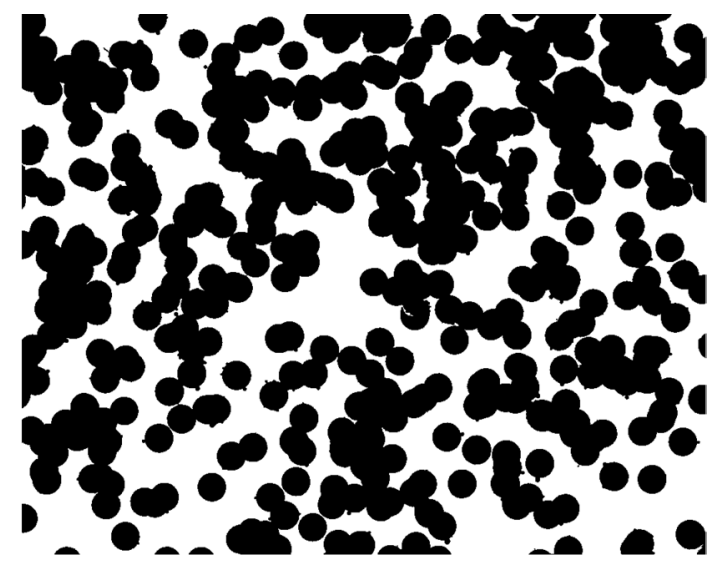

(a) Example of an experimental binarized image

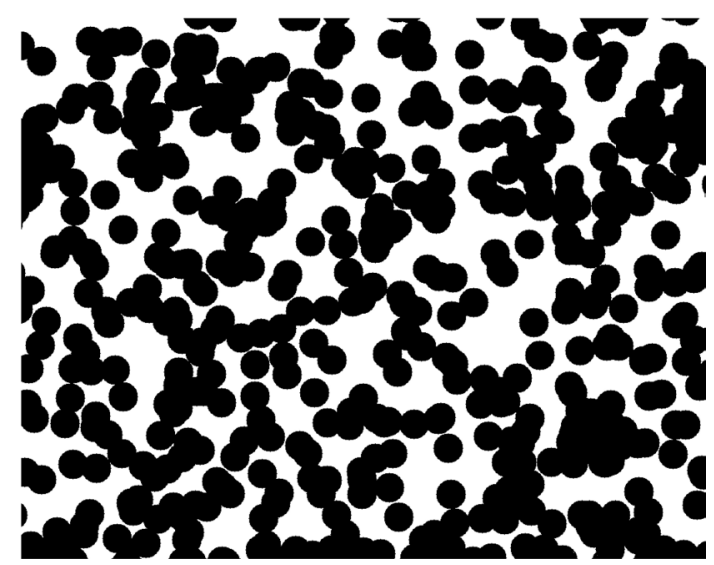

(b) Example of a modeled image

Fig. 11. Qualitative comparison between an synthetic image coming from the calibrated model (for $\alpha=$ $1 / 2)$ and an experimental image of the monodisperse population of PMMA spheres.

\section{DISCUSSION}

In the previous section, the first three experiments conducted on the synthetic images enabled to validate the calibration routine proposed. They moreover enabled to quantify the sensitivity of the procedure regarding the volume fraction of the population of spheres, and the initial solution of the optimization routine. The last test case was designed to evaluate the behavior of the algorithm when the radius distribution of the particles is not unimodal.

In the first case study, we observe that the proposed optimization routine performs well for a weight parameter $\alpha=0$ (resp. $\alpha=1 / 2$ ): the maximum relative error for the intensity is less than $5 \%$ (resp. 9.5\%), 3.5\% (resp. 5.5\%) for the mean and $2 \%$ for the standard deviation of the radius distribution. For $\alpha=1$, with a relative around $9 \%$, the estimation of the standard deviation is rather coarse, hence the deviation for the opening function. This result 


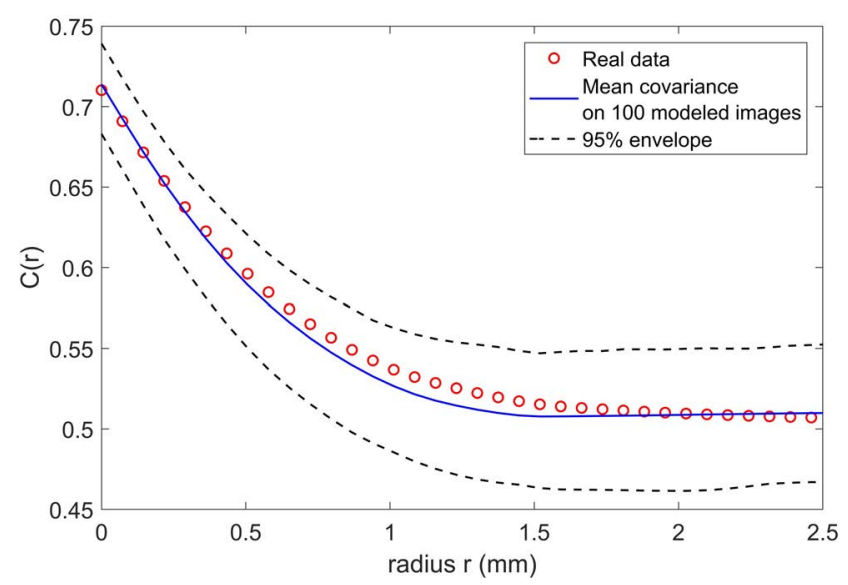

(a) Fitted covariance

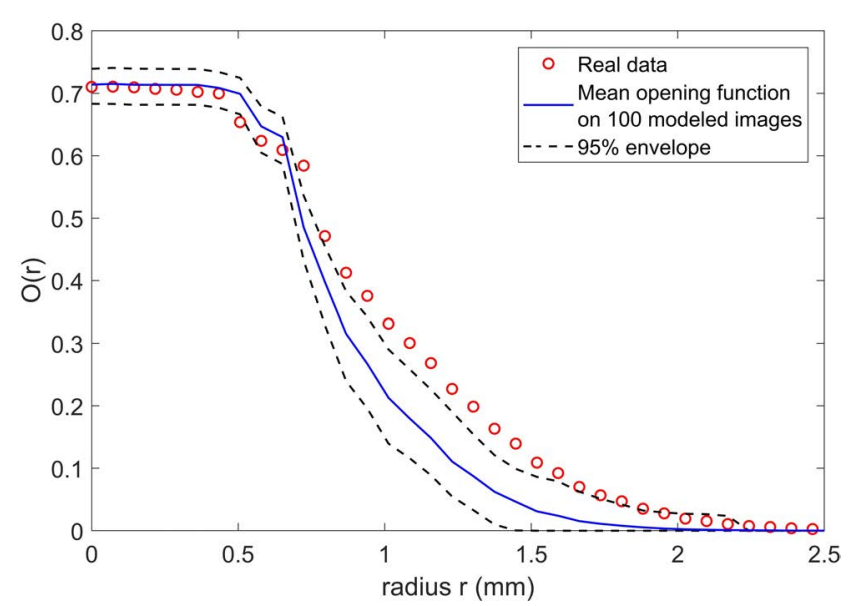

(b) Fitted opening function

Fig. 12. Fitted characteristics for the two-phase flow where the dispersed phase is a controlled population of bi-dispersed PMMA spheres. The real data characteristics are plotted in red circles. The mean characteristics of the fitted model on a set of 100 images are represented in blue lines and its 95\% envelope in black dashed-lines.

emphasizes that the opening function captures most of the geometrical information. Changing the volume fraction has little effect on the fitting parameters, thus highlighting a weak sensitivity to the $V_{V}$ value. There are two exceptions: i) when very few information is present on the images $\left(V_{V}=1 \%\right)$, and ii) when the images are almost black (more than $90 \%$ of the images is covered). Conversely, the accuracy of the statistical inference depends on the initial solution, and on how far it is from the true solution. Indeed, as shown in Table 2, if the initial solution deviates more than $20 \%$ from the real values of intensity and mean parameter, and $40 \%$ from the standard deviation, then significant deviations can be expected (for at least one of the parameters). The numerical test regarding

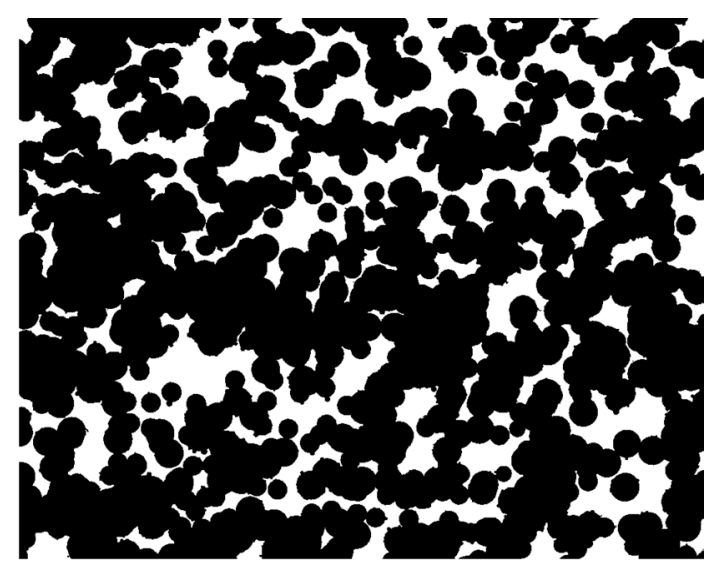

(a) Example of an experimental binarized image

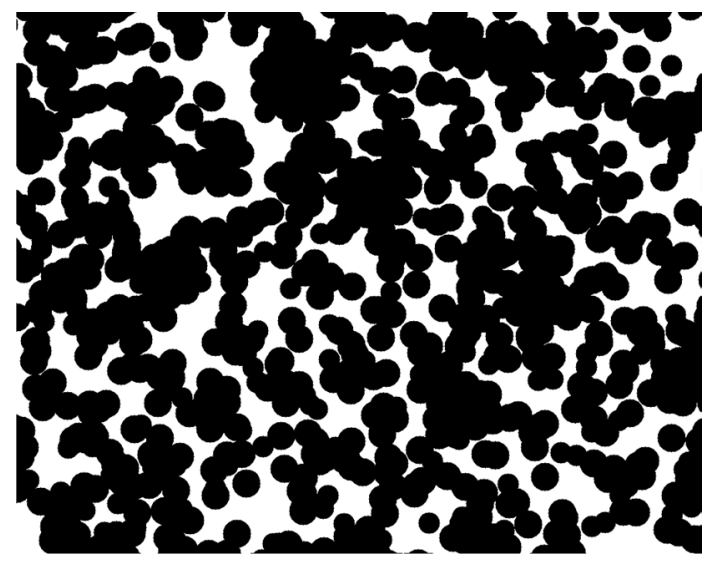

(b) Example of a modeled image

Fig. 13. Qualitative comparison between a synthetic image coming from the calibrated model (for $\alpha=$ $1 / 2$ ) and an experimental image of the bidisperse population of PMMA balls.

bimodal populations, demonstrates the efficiency of the proposed optimization procedure when mixtures of particles exhibiting different radii are considered. The results highlight that the geometrical information is well retrieved regardless of whether the proportions of each sub-population is known or whether the two radius are observed. Nevertheless, the results are less reliable if the weight parameter $\alpha$ is not equal to 0 . This indicates that the opening function better compensates for the lack of information due to the overlapping.

At last, validation on real images of two simulated two-phase flows emphasizes the relevance of the model. On the one hand, the test on a monodisperse population of PMMA spheres demonstrates that the information of interest (the radius and the mean number of particles per unit volume) is quite well retrieved. However, while the covariance is well fitted, a significant deviation on the right part of the opening 
function is noticed. It has been observed during the experiments that the PMMA spheres had a tendency to stick to each other when touching, leading to the appearance of larger aggregates. This typical and not expected behavior cannot be reproduced by the proposed model, hence explaining the higher value of the real opening function predicted for the larger radius $r$. On the other hand, the model deviation is more evidenced in the experiment with the bidisperse population of PMMA spheres. The best results in terms of covariance and opening function fitting are obtained for $\alpha=1 / 2$ where $\hat{\lambda}_{a t}=0.0179, \hat{r}_{1}=0.567$, $\hat{r}_{2}=0.742$ and $\beta_{a t}=0.41$, corresponding to a relative error for $r_{1}$ of $13 \%$ and for $r_{2}$ of $1 \%$. Moreover, the intensity is underestimated by about $22 \%$. It is difficult to conclude whether this underestimation is due to the violation of the uniformity assumption on which is based the calculation of $\lambda_{\text {real }}$ or to hidden information that cannot be retrieved. Besides, keeping the covariance in the objective function enables to control the model deviation, while still obtaining reasonable results for the radius.

The numerical experiments have shown that the weight parameter $\alpha$ can influence the accuracy of the fitted parameters. As a rule of thumb, we advise to always consider the opening function, as the driven functional characteristics as it contains most of the geometrical information. Moreover, at $r=0$ its value corresponds to the volume fraction of the random set and then enables to control the intensity $\lambda$. As an a posteriori check, the adjustment on other functional characteristics such as the spherical contact distribution function or the morphological functions can also be considered for model validation.

Finally, it is worth noting that the construction of the initial solution is based on fitting the silhouette images with a boolean model of disks. However, the images of the two-phase flows should not be strictly modeled by a boolean model because 3D interactions between the particles exist, that can be retrieved in the 2D images. Statistical tests of the boolean model hypothesis, such as proposed in Mrkvička (2009), should confirm this conjecture.

\section{CONCLUSION}

In this study, we have demonstrated that 3D modeling is a new and promising approach for characterizing two-phase flows. The validation on synthetic images proves its reliability, and the application on real suspensions of, possibly polydispersed, PMMA spheres illustrates its relevance in the case of a dense flow. The proposed method has several advantages: i) it enables to model both the actual (i.e., 3D) particles population in the flow and their 2D silhouette images, ii) the image acquisition process is taken into consideration, iii) important physical characteristics of the particles can be derived from the proposed model, iv) realistic volume fraction can be handled without significant loss of precision, v) at last it is easy to implement, thus convenient for practical/engineering applications.

Although the case of spheres may not seem the most relevant for correlating 3D properties from 2D images, this first step was of paramount importance for the validation of the implemented mathematical transformations and the proposed interaction function. Considering spheres also facilitated the realization of experimental validation. Given the good performances exhibited by the proposed methodology, while applied to spheres, two main improvements are currently being developed in order to enhance both the domain of application and the flexibility of the model regarding typical dispersed systems.

The first one is aimed at generalizing the model to non-spherical particles, in particular to ellipsoids frequently encountered in bubbly flows (de Langlard $e t$ al., 2018). The difficulty lies in the construction of the initial solution, as the relationships between the 2D and 3D geometrical characteristics for random ellipsoids are not straightforward.

The second improvement is aimed at modeling more complex interactions between the particles. This will require a more general deletion process. Based on the recent work of Teichmann et al. (2013), the deletion probability $p$ can depend on the value of its mark, rather than removing the point surely. This generalization may be used to model phenomena such as coalescence or breakage.

\section{ACKNOWLEDGEMENT}

This work was supported by the Nuclear Energy Division of CEA (program DISN/PRATA).

\section{REFERENCES}

Audet C, Dennis JE (2002). Analysis of generalized pattern searches. SIAM J Optimiz 13:889-903.

Arns CH, Knackstedt MA, Mecke KR (2003). Reconstructing complex materials via effective grain shapes. Phys Rev Lett 91:215506.

Baddeley A, Jensen EBV (2004). Stereology for statisticians. New York: Chapman \& Hall/CRC.

Biswal B, Øren P E, Held R J, Bakke S, Hilfer R (2011). Modeling of multiscale porous media. Image Anal Stereol 28:23-34. 
Chiu SN, Stoyan D, Kendall W, Mecke J (2013). Stochastic geometry and its applications, 3rd Ed. Chichester: Wiley.

Daley DJ, Vere-Jones D (2003). An introduction to the theory of point processes. Vol I: Elementary theory and methods. New York: Springer.

Davidon WC (1991). Variable metric method for minimization. SIAM J Optimiz 1:1-17.

de Langlard M, Al-Saddik H, Charton S, Debayle J, Lamadie F (2018). An efficiency improved recognition algorithm for highly overlapping ellipses: application to dense bubbly flows. Pattern Recogn Lett 101:88-95.

Dereudre D, Lavancier F, Helisová K (2014). Estimation of the intensity parameter of the germ-grain Quermassinteraction model when the number of germs is not observed. Scand J Stat 41:809-29.

Descombes X (2011). Application de la géométrie stochastique à l'analyse d'images. Paris: HermèsLavoisier

Diggle P (1981). Binary mosaics and the spatial pattern of heather. Biometrics 37:531-9.

Emery X, Kracht W, Egana A (2012). Using two-point set statistics to estimate the diameter distribution in Boolean models with circular grains. Math Geosci 44:805-22.

Hall P (1985). Counting methods for inference in binary mosaics. Biometrics 41:1049-52.

Illian J, Penttinen A, Stoyan H, Stoyan D (2008). Statistical analysis and modelling of spatial point patterns. Chichester: Wiley.

Kracht W, Emery X, Paredes C (2013). A stochastic approach for measuring bubble size distribution via image analysis. Int J Miner Process 121:6-11.

Kubínová L, Radochová B, Lhotáková Z, Kubinová Z, Albrechtová J (2017). Stereology, and unbiased methodological approach to study plant anatomy and cytology: past, present and future. Image Anal Stereol 36:187-205.

Lau YM, Deen NG, Kuipers JAM (2013). Development of an image measurement technique for size distribution in dense bubbly flows. Chem Eng Sci 94:20-9.
Maaß S, Wollny S, Voigt A, Kraume M (2011). Experimental comparison of measurement techniques for drop size distributions in liquid/liquid dispersions. Exp Fluids 50:259-69.

Månsson M, Rudemo M (1960). Random patterns of nonoverlapping convex grains. Adv Appl Probab $34: 718-38$

Matérn B (1960). Spatial variation: stochastic models and their application to some problems in forest surveys and other sampling investigations. Statens skogsforskningsinstitut.

Matheron G (1972). Ensembles alétoires et géométrie intégrale. Les cahiers du centre de morphologie mathématiques de Fontainebleau.

Mrkvička T, Rataj J (2009). On estimation of intrinsic volume densities of stationary random closed sets via parallel sets in the plane. Kybernetika 45:931-45.

Mrkvička T (2009). On testing of general random closed set model hypothesis. Kybernetika 45:293-308.

Mrkvička T, Mattfeldt T (2011). Testing histological images of mammary tissues on compatibility with the boolean model of random sets. Image Anal Stereol 30:11-8.

Muthukannan K, Latha P (2015). A PSO model for disease pattern detection on leaf surfaces. Image Anal Stereol 34:209-16.

Nagata T (2000). Three-dimensional observations on thick biological specimens by high voltage electron microscopy. Image Anal Stereol 19:51-6.

Ohser J, Schladitz K (2009). 3D images of materials structures: processing and analysis. Weinheim: Wiley $\mathrm{VCH}$.

Stoyan D, Stoyan H (1985). On one of Matérn's hardcore point process models. Math Nachr 122:205-14.

Teichmann J, Ballani F, van den Boogaart K.G (2013). Generalizations of Mátern hard-core point processes. Spat Stat 3:33-53.

Weil W (1988). Expectation formulas and isoperimetric properties for non-isotropic Boolean models. J Microsc 151:235-45.

Zafari S, Eerola T, Sampo J (2015). Segmentation of overlapping elliptical objects in silhouette images. IEEE T Image Process 24:5942-52. 